\title{
A case series of eight scoliosis patients undergone pedicle screw placement with freehand technique: study for safety and accuracy
}

Komang Agung Irianto, Marquee Kenny Tumbelaka

pISSN: 0853-1773 • elSSN: 2252-8083 https://doi.org/10.13181/mji.cr.191978 Med J Indones. 2020;29:222-7

Received: May 4, 2017

Accepted: August 20, 2019

Authors' affiliations:

Department of Orthopaedic and Traumatology, Faculty of Medicine, Universitas Airlangga, Dr. Soetomo Hospital, Surabaya, Indonesia

\section{Corresponding author:}

Komang Agung Irianto

Department Orthopaedic and

Traumatology, Dr. Soetomo Hospital,

Jalan Prof. Dr. Moestopo No. 6-8,

Surabaya 60286, Indonesia

Tel/Fax: +62-31-5020406

E-mail: komang168@yahoo.com

\begin{abstract}
BACKGROUND Pedicle screws and rods are routinely inserted in the vertebrae thoracic to lumbar in scoliosis reconstruction surgery to gain deformity correction and fusion. Intraoperative imaging is often needed to ensure accuracy, but surgical time will be longer and there will be more radiation exposure. Meanwhile, freehand technique is accepted as safe. This study was aimed to evaluate the accuracy of screw insertion in the freehand technique used in adolescent scoliosis surgery.
\end{abstract}

METHODS This case series evaluated a total of 127 pedicle screws inserted using the freehand technique in 8 out of 28 adolescent scoliosis patients from 2011 to 2016 whom agreed for computed tomography (CT) scan follow-up from 2011 to 2016 in Dr. Seotomo Hospital, Surabaya. The accuracy and safety of the freehand technique were evaluated postoperatively in each patient using a CT scan. A successful screw was considered accurate if it was within the vertebral body and inside the safety zone (within the limit of 2-4 $\mathrm{mm}$ to the medial or lateral side of the vertebral body.

RESULTS Of the 127 screws inserted, 106 (83.5\%) were accurately placed, and 110 $(86.6 \%)$ were within the safe zone. According to the level of the spine, in the upper and middle thoracic spine, the inserted screws were accurate in $69.4 \%$ and $74.9 \%$ within the safety zone, whereas in the lower thoracic and lumbar spine, $94.0 \%$ and $95.0 \%$ within the safety zone.

CONCLUSIONS The freehand technique in scoliosis reconstructive surgery performed by experienced surgeons is accurate and safe.

KEYWORDS pedicle screws, scoliosis
Three compartment fixation using pedicle screws in the posterior spinal element remains popular as a derotation, translation, distraction, and compression method in scoliosis reconstruction. This system allows accurate correction at each segment in all three planes. ${ }^{1}$ Pedicle screws have several advantages, such as overall constructive rigidity, stability necessary for spinal arthrodesis, and improvement of deformity correction due to its three-column control over the spinal elements. ${ }^{2}$ Pedicle screw instrumentation obviates the need to place instrumentation within the spinal canal, thus lessening the risk of neural injury. ${ }^{1}$

Olsewski et $\mathrm{al}^{3}$ claimed that lumbar vertebrae morphometry is suitable and able to safely accommodate pedicle screws instrumentation. Vaccaro et $\mathrm{al}^{4}$ found that pedicle in thoracic vertebrae, despite the smaller size, was also suitable for pedicle screw instrumentation. The situation changes in the case of a deformed spine, as in adolescence scoliosis, where the transverse process may be abnormal in 
shape and size due to rotation and wedging. ${ }^{5}$ Surgeons have to remember that morphometry and anatomical landmarks can only provide a rough guideline. ${ }^{3}$ The base of the superior facet is a reliable landmark in identifying the pedicle, even in deformed spines. ${ }^{5}$ Preoperative assessment to evaluate the size of the pedicles, angle, and depth of the screw is necessary to alleviate the surgeon's confidence in properly placing the entry point. ${ }^{1,3,5}$

Malposition of a pedicle screw could violate the spinal cortex, causing neurological and vascular complications. ${ }^{1,2}$ Owing to financial reasons, a lack of equipment, and radiation safety for medical personnel and patient, our institution adopted the freehand technique. The pedicle screw insertion of thoracic and lumbar spine in this technique is guided by anatomical marker only instead of fluoroscopy. Its safety has been proven by several published studies. ${ }^{1,2}$ In our center, the surgeries were performed by senior spine surgeons. Although our institution has never encountered any neurological and vascular complications of screw violation cases that could jeopardize the patients' safety, we understand the necessity of evaluating a proper screw insertion. This study was aimed to evaluate the accuracy and safety of freehand technique in scoliosis patients.

\section{METHODS}

This is a case series that evaluated the accuracy and safety of a total of 127 pedicle screws inserted using the freehand technique in 8 scoliosis patients by a single experienced orthopedic spine surgeon in Dr. Soetomo Hospital, Surabaya. The study has been approved by our internal institutional board (520/Panke.KKE/ VIII/2017). We evaluated 8 out of 28 adolescent scoliosis patients from 2011 to 2016, consisting of 7 females and 1 male, who agreed to participate in the study went through computed tomography (CT) scan, which was performed during follow-up of 6-40 months with an average of 17 months.

A total of 127 screws were inserted: 29 in the upper thoracic ( $\left.\mathrm{T}_{1-\mathrm{T}} 4\right), 27$ in the midthoracic ( $\mathrm{T}_{5}-$ T8), 25 in the lower thoracic (T9-T12), and 46 in the lumbar vertebra. All screws were inserted using the freehand technique. All $\mathrm{CT}$ scan results were reviewed by radiologist (Figure 1). Screw accuracy was independently evaluated according to the Heary classification. ${ }^{6}$
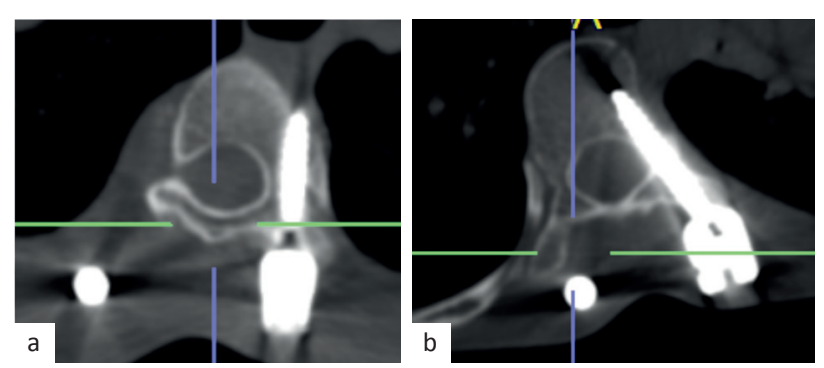

Figure 1. Example of (a) breaching screw of the lateral wall of the pedicle and (b) accurate screw in the thoracic spine

Medial or lateral screw penetration of the pedicle was categorized into grade o (fully contained within the pedicle), grade 1 (penetration $<2 \mathrm{~mm}$ ), grade 2 (penetration 2.1-4.0 mm), grade 3 (penetration 4.1-6.0 $\mathrm{mm}$ ), and grade 4 (penetration $>6 \mathrm{~mm}$ ). The screw penetrations anterior to the vertebral body were also measured using the same method. The screws displaced medially by $<2 \mathrm{~mm}$ and laterally by $<4 \mathrm{~mm}$ from pedicle were considered as within the safe zone. ${ }^{6}$ The accuracy between the upper, middle, and lower thoracic and lumbar spine was also analyzed.

\section{The freehand technique of scoliosis vertebrae}

The approach to scoliosis surgery is posterior. The freehand technique started by making an incision and exposure until the tip of the bilateral transverse processes is seen. The surgeon marks the entry point at the base of the superior articular process (the base
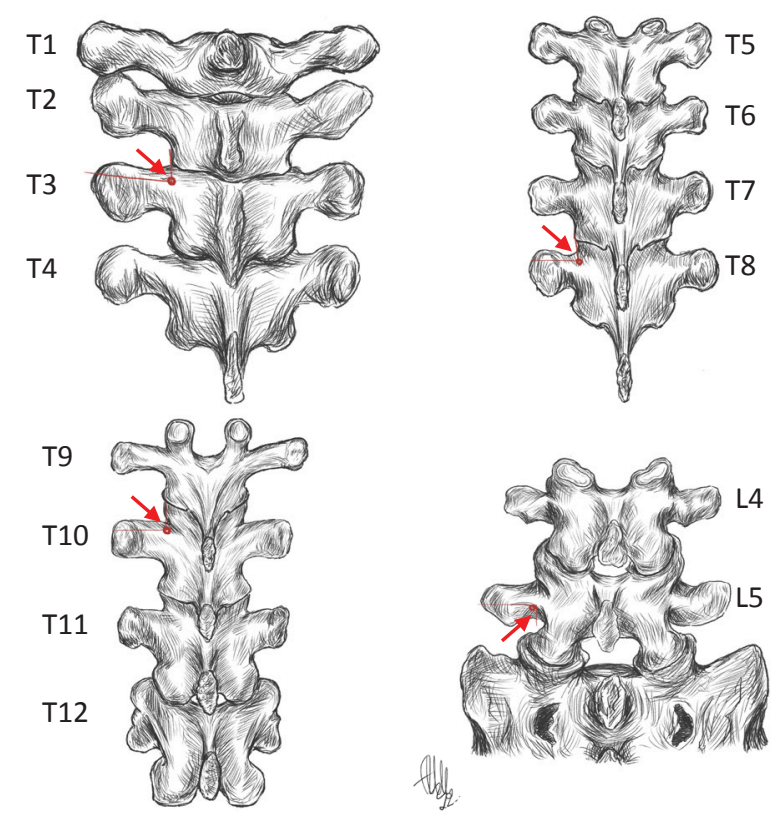

Figure 2. Red dots and red arrows show the pedicle screw entry point for upper thoracic (T1-T4), midthoracic ( $\mathrm{T}_{5}-$ T8), lower thoracic (T9-T12), and lumbar vertebrae (L1-L5) (Illustration by author). T=thoracic; L=lumbar 
of the superior facet), by the junction of the lateral onethird and medial two-thirds, making an ideal pedicle entry point. ${ }^{7}$ The curve scoliosis vertebrae are different from normal vertebrae in an educational cadaver. The starting point is different between the upper, middle, lower thoracic, and lumbar vertebra (Figure 2). In thoracic 1 and 2, the starting point is at the junction of the bisected transverse process and lamina at the lateral pars articularis. Thoracic 7 and 9 have the most medial starting point, on the junction of proximal edge of the transverse process and lamina, where it meets the lamina and superior facet, lateral to the midportion of the base of the superior articular process. For the lower thoracic and lumbar, the starting point is on the junction of the bisected transverse process and lamina, from the medial to the lateral aspect of the pars articularis. 7,8

The screw insertion begins on the concave side of the neutral and most distally instrumented vertebra, one level below the lower end vertebrae, then gradually moves to the proximal edge. The starting point guides surgeons to the second screw position according to rotation direction, which is shown by a Kirschner wire marker in the first starting point. The proximal screw direction is guided by the distal screw coming way up through proximity to the first horizontal plane, continuing upward from further neutral vertebrae. Knable was used to draw a hole for the awl to get through. The awl is pushed in by wiggling it. The tip will go down the cancellous portion of the pedicle until it touches the center, proofed by oozing of the blood of the central artery. The awl is used to create a posterior cortical breach, and then, the surgeon probes it laterally as a safety measure to avoid medial wall perforation. After inserting the tip approximately $15-20 \mathrm{~mm}$, the tip is turned to face medial, using the feeler to touch the hole, making sure that the medial, proximal, distal, and lateral borders are not perforated. The depth average of the awl is $40-45 \mathrm{~mm}$ in the lumbar, $30-40 \mathrm{~mm}$ in the lower thoracic, 25-30 $\mathrm{mm}$ in the midthoracic, and 20$25 \mathrm{~mm}$ in the upper thoracic regions. After the awl is removed, the tract is visualized to make sure that only blood is coming out and not the cerebrospinal fluid. Then, the surgeon will do the tapping and repalpating before inserting the screw. ${ }^{9,10}$

\section{RESULTS}

Of the 127 total screws inserted in eight patients (Table 1), 106 (83.5\%) pedicle screws were accurately placed, and 21 were all laterally displaced, devoid of the medial, superior, inferior, and anterior violations. Only 10 screws (7.9\%) were displaced to more than 6 $\mathrm{mm}$ laterally, but none has injured vital essential organs or tissues, caused neurologic complications, or needed further screw repositioning (Table 2).

The most inaccurate screw placement was in the upper and midthoracic regions where the range are $65.5-74.1 \%$. While the safest and accurate screw placement were in the lower thoracic and lumbar spine (92.0-95.7\%). The accuracy and safety of the freehand technique for scoliosis reconstruction surgery in the thoracolumbar region is higher than in the upper and midthoracic regions (Figure 3).

\section{DISCUSSION}

The goal of scoliosis reconstruction surgery, albeit the causes (adolescent idiopathic scoliosis [AIS], Marfan syndrome, or neurofibromatosis type 1 ), is a safe

Table 1. Characteristics of subject

\begin{tabular}{llcccc}
\hline No. & Sex & Age (years) & Disease & Screws inserted & Displaced screws \\
\hline 1 & Female & 13 & AIS & 18 & T2, T7, T12 \\
2 & Female & 19 & AIS & 14 & T3, T7 \\
\hline 3 & Female & 13 & AIS & 20 & T2, T7, L3 \\
4 & Female & 12 & Marfan syndrome & 15 & T1, T8 \\
\hline 5 & Female & 15 & Marfan syndrome & 16 & T2, T4 \\
\hline 6 & Female & 17 & Marfan syndrome & 16 & T2, T4, L1 \\
7 & Female & 12 & Marfan syndrome & 17 & T2, T4, T7 \\
8 & Male & 20 & Neurofibromatosis type 1 & T5, T7, T11 \\
\hline
\end{tabular}

AIS=adolescent idiopathic scoliosis

The bold number is the level where the screw displaced $>6 \mathrm{~mm}$

mji.ui.ac.id 
Table 2. Pedicle screw insertion displacement on the thoracic and lumbar regions

\begin{tabular}{|c|c|c|c|c|}
\hline Level & $\begin{array}{l}\text { Accurate screws } \\
\qquad(N=106)\end{array}$ & $\begin{array}{l}\text { Medially/laterally } \\
\text { displaced }(\mathrm{N}=21)\end{array}$ & $\begin{array}{l}\text { Screw within the } \\
\text { safe zone }(N=127)\end{array}$ & $\begin{array}{c}\text { Total screws } \\
\text { inserted }(N=127)\end{array}$ \\
\hline $\mathrm{T} 1$ & 5 & 2 & 5 & 7 \\
\hline $\mathrm{T} 2$ & 3 & 4 & 3 & 7 \\
\hline T3 & 8 & 1 & 8 & 9 \\
\hline T4 & 3 & 3 & 4 & 6 \\
\hline UT & 19 & 10 & 20 & 29 \\
\hline T5 & 5 & 1 & 5 & 6 \\
\hline T6 & 6 & 1 & 7 & 7 \\
\hline T7 & 4 & 4 & 5 & 8 \\
\hline T8 & 5 & 1 & 5 & 6 \\
\hline MT & 20 & 7 & 22 & 27 \\
\hline T9 & 4 & 0 & 4 & 4 \\
\hline T10 & 8 & 0 & 8 & 8 \\
\hline T11 & 3 & 1 & 3 & 4 \\
\hline T12 & 8 & 1 & 8 & 9 \\
\hline LT & 23 & 2 & 23 & 25 \\
\hline L1 & 10 & 1 & 11 & 11 \\
\hline L2 & 10 & 0 & 10 & 10 \\
\hline L3 & 10 & 1 & 10 & 11 \\
\hline L4 & 13 & 0 & 13 & 13 \\
\hline L5 & 1 & 0 & 1 & 1 \\
\hline L & 44 & 2 & 45 & 46 \\
\hline
\end{tabular}

T=thoracic; UT=upper thoracic; $M T=$ midthoracic; $L T=$ =lower thoracic; $L=l u m b a r$ correction of the deformity with spinal instrumentation and solid fusion. ${ }^{5}$ Pedicle screw instrumentation has been proven to be reliable and effective in the surgical management of scoliosis. ${ }^{1,5}$ Freehand technique in an experienced surgeon simplifies the process of pedicle screw insertion and speeds up surgical procedure, and

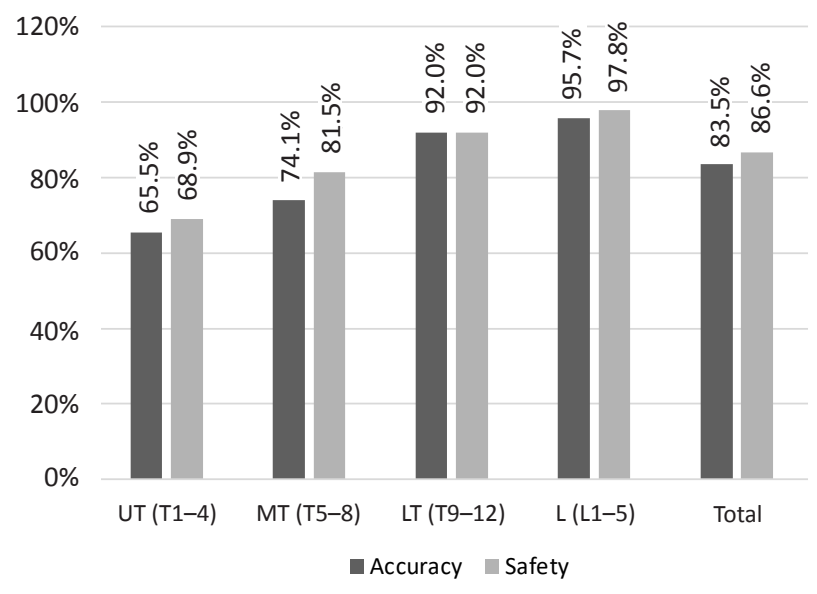

Figure 3. Accuracy and safety of pedicle screw insertion. $\mathrm{T}=$ thoracic; UT=upper thoracic; MT=midthoracic; $\mathrm{LT}=$ =lower thoracic; L=lumbar there is no risk of radiation exposure..$^{1,2,8}$ In a metaanalysis, Tian et al ${ }^{1}$ investigated the accuracy of pedicle screw insertion in various spinal diseases with various methods and they found that navigated technique was more accurate along with similar clinical outcomes. AIS patients are mostly teenager and unnecessary radiation exposure during surgery need to be avoided for their good quality of life in the future. Gebhard et al ${ }^{11}$ reported that although computer-assisted surgery reduced the exposure time and doses of intraoperative radiation, the advanced $C_{3} D$ fluoroscopy-based navigation still spread $152 \mathrm{mGy}$.

In this study, we analyzed the accuracy and safety of pedicle screw instrumentation in adolescent scoliosis using the freehand technique after an average of 17 months follow-up, which shows that all patients were well corrected without any neurologic or vascular complications. The accuracy (83.5\%) and safe zone rates $(86.6 \%)$ were considered excellent compared with other studies that used a cadaver model.4,7 Other in vivo study in the patient using $2 \mathrm{D}$ image guidance, the accuracy rate was reported $84.3 \% .^{12}$ The accuracy 
and safety in the lower thoracic and lumbar spine are higher than in the upper thoracic and midthoracic regions. This finding is concurrent with other studies and provides a good evidence base for the freehand technique. ${ }^{6,7,9,10}$

Su et $\mathrm{al}^{5}$ reported the overall accuracy of lumbar pedicle screw placement was 93.5-99.1\% with different entry points of pedicle screws. Margel's technique was used in many centers whether for computed guided, navigation, or freehand, to approach the entry point of lumbar spine at the junction of the lateral border of the superior articular process and the bisector of the transverse process.5,7,9 Similarly, this study reported 92-97\% of accuracy in lumbar pedicle screws among scoliosis cases. A report for the thoracic region on scoliosis patients also showed a similar result. Our study showed 20\% (lower thoracic) to 32\% (upper thoracic) lateral breaching screw compared with $25 \%$ to $30 \%$ in the largest published clinical series to date. ${ }^{13}$ Because of the variability in these individual parameters, "freehand" pedicle instrumentation in the upper and midthoracic spine based exclusively on anatomic landmarks is considered to be imprecise and eventually led to unacceptable rates of misplaced screws. ${ }^{6,7,9,10}$ Clinical and cadaveric studies have shown that about $15 \%$ to $50 \%$ of thoracic screws placed using the "freehand" technique may violate the pedicular cortex, although clinical and follow-up showed that the increased rates of radiographic cortical violations did not necessarily correlate with poorer clinical outcomes. . $^{1,6,8,9,14}$

The freehand technique used in our institution is not similar to Kim's technique. ${ }^{7}$ We did not use a guided probe, which provides "beep" sound whenever it touches the cortex wall before it penetrates it. Suess and Schomacher ${ }^{15}$ tried an electrical conductivity measurement device to control the possibility of screw breach. Instead, the funnel technique was used by wiggling the awl to the center of the cortex.1,3,4 The funnel technique has a risk of screw loosening as a consequence of excessive loss of cancellous bone, pedicle fracture, and long operation time..$^{1,3,4}$ The risk for excessive bone loss could be avoided by putting the screw laterally and avoiding creating a hole, which is larger than the screw using the knable. In this study, the problem was never encountered since the pedicle was felt using the root dissector and palpated along with the wiggling probe. Oh et al $^{7}$ reported a similar displaced rate to the lateral side. According to several studies, the medial wall of the pedicle is thicker than the lateral wall. ${ }^{6,7,9}$ In scoliosis, the lateral medial diameter of the pedicle on the concave side is narrower than on the convex side, especially in the apex. Our study showed that grade 4 (penetration $>6 \mathrm{~mm}$ ) to lateral were more pronounced at $\mathrm{T}_{2}$ and $\mathrm{T} 7$ levels (Table 2 ). These breaching screws were found in patients with AIS and Marfan syndrome, and they were the last screws to be inserted ( $\left.\mathrm{T}_{2}\right)$ and at the apex of the curve ( $\mathrm{T}_{7}$ ) (Table 1). For the patient with neurofibromatosis type 1 , the breaching screws were at the apex (T11) and at the last inserted screw ( $\mathrm{T}_{5}$ ), there was no neurological sequel but this rare condition needs to be confirmed with more example cases.

The limitation of this case series was a small subjects number and all the subjects were undergone surgeries only by a single experienced orthopedic surgeon (KAIS). More studies should be performed to assess other parameters that may affect the safety and accuracy of screw placement in scoliosis patients in the future. In conclusion, the freehand technique in scoliosis reconstructive surgery performed by an experienced surgeon is accurate and safe.

\section{Conflict of Interest}

The authors affirm no conflict of interest in this study.

\section{Acknowledgment}

The authors would like to thank all residents and patients involved in this study, Sindrawati Oen for editing, and Nabila Ananda Kloping for drawing the illustration.

Funding Sources

None.

\section{REFERENCES}

1. Tian NF, Huang QS, Zhou P, Zhou Y, Wu RK, Lou Y, et al. Pedicle screw insertion accuracy with different assisted methods: a systematic review and meta-analysis of comparative studies. Eur Spine J. 2011;20(6):846-59.

2. Vijayeswaran N, Venkatesh R, Murugesan G, Balamurugan S, Indunesh K, Pradeep TT. Is freehand technique of pedicle screw insertion in thoracolumbal spine safe and accurate? Assessment of 250 screws. J Neurosci Rural Pract. 2019;10(2):256-60.

3. Olsewski JM, Simmons EH, Kallen FC, Mendel FC, Severin, CM, Berens DL. Morphometry of the lumbar spine: anatomical perspectives related to transpedicular fixation. J Bone Joint Surg Am. 1990;72(4):541-9.

4. Vaccaro AR, Rizzolo SJ, Balderston RA, Allardyce TJ, Garfin SR, Dolinskas $C$, et al. Placement of pedicle screws in the thoracic spine. Part II: An anatomical and radiographic assessment. J Bone Joint Surg Am. 1995;77(8):1200-6.

5. Su P, Zhang W, Peng Y, Liang A, Du K, Huang D. Use of computed tomographic reconstruction to establish the ideal entry point for pedicle screws in idiopathic scoliosis. Eur Spine J. 2012;21(1):23-30.

6. Rivkin MA, Okun JF, Yocom SS. Novel free-hand T1 pedicle screw method: review of 44 consecutive cases. J Neurosci Rural Pract. 2014;5(4):349-54. 
7. Oh CH, Yoon SH, Kim YJ, Hyun D, Park HC. Technical report of free hand pedicle screw placement using the entry points with junction of proximal edge of transverse process and lamina in lumbar spine: analysis of 2601 consecutive screws. Korean $J$ Spine. 2013;10(1):7-13.

8. Li J, Zhao H, Xie H, Yu L, Wei J, Zong M, et al. A new free-hand pedicle screw placement technique with reference to the supraspinatus ligament. J Biomed Res. 2014;28(1):64-70.

9. Chan A, Parent E, Narvacan K, San C, Lou E. Intraoperative image guidance compared with free-hand methods in adolescent idiopathic scoliosis posterior spinal surgery: a systematic review on screw-related complications and breach rates. Spine J. 2017;17(9):1215-29.

10. Avila MJ, Baaj AA. Freehand thoracic pedicle screw placement: review of existing strategies and step by step guide using uniform landmark for all levels. Cureus. 2016;8(2):e501.

11. Gebhard FT, Kraus MD, Schneider E, Liener UC, Kinzl L, Arand. Does computer-assisted spine surgery reduce intraoperative radiation doses? Spine. 2006;31(17):2024-7.

12. Mason A, Paulsen R, Babuska JM, Rajpal S, Burneikiene S, Nelson EL, et al. The accuracy of pedicle screw placement using intraoperative image guidance systems. J Neurosurg Spine. 2014;20(2):196-203.

13. de Marco FA, Risso-Neto MI, Cavali PTM, Sussi MA, Pasqualini W, Landim E. Placement analysis of thoracic and lumbar pedicle screws inserted under anatomic and radioscopic parameters. Columna. 2008;7:1-7.

14. Parker SL, Amin AG, Santiago-Dieppa D, Liauw JA, Bydon A, Sciubba DM, et.al. Incidence and clinical significance of vascular encroachment resulting from freehand placement of pedicle screws in the thoracic and lumbar spine: analysis of 6816 consecutive screws. Spine. 2014;39(8):683-7.

15. Suess $O$, Schomacher M. Control of pedicle screw placement with an electrical conductivity measurement device: initial evaluation in the thoracic and lumbar spine. Adv Med. 2016;2016:4296294. 